\title{
Impact of Educational Experience on Attitudes Toward Mental Illness and Career Choice
}

\author{
Richard Balon $^{1}$ • Mary K. Morreale ${ }^{1}$ - Anthony P. S. Guerrero ${ }^{2}$ - Eugene V. Beresin ${ }^{3}$. \\ Adam Brenner $^{4}$ • John H. Coverdale ${ }^{5}$ - Alan K. Louie ${ }^{6}$ - Laura Weiss Roberts ${ }^{6}$
}

Received: 9 February 2017 / Accepted: 12 February 2017 / Published online: 28 February 2017

(C) Academic Psychiatry 2017

The impact of educational experiences on medical students' attitudes towards psychiatry has been studied for decades [1-4]. What has been elucidated from research into this topic thus far is that the psychiatric clerkship can strongly influence students' views of our specialty and that this influence occurs mostly in a positive direction. Three articles in the June 2017 issue of Academic Psychiatry [5-7] add interesting data to the extant literature on the subject. These articles further address the impact of educational experiences on attitudes toward mental illness, how students perceive the efficacy of psychiatric treatments, and the complicated relationship between attitudes toward our specialty and the choice of mental health professions as a career goal.

Itzhaki and colleagues [5] questioned 101 nursing students at four academic nursing schools in Israel regarding changes in attitudes and intention to work with patients with mental illness before and after a planned educational intervention. The intervention consisted of five identical components at all four institutions: (1) a one-on-one encounter with two people living with either schizophrenia or a mood disorder who spoke about their life and illness; (2) an encounter with a woman who suffered from anorexia nervosa as a teenager;

Richard Balon

rbalon@wayne.edu

Wayne State University, Detroit, MI, USA

2 University of Hawai'i John A. Burns School of Medicine, Honolulu, HI, USA

3 Harvard Medical School, Boston, MA, USA

4 University of Texas Southwestern Medical Center, Dallas, TX, USA

5 Baylor College of Medicine, Houston, TX, USA

6 Stanford University, Stanford, CA, USA
(3) the viewing and subsequent discussion of a 50-min film that told the story of a woman who coped with lifelong mental illness, experienced inpatient and community-based treatments, and ultimately earned a $\mathrm{PhD}$ in psychology, with a discussion that followed; (4) two simulations (one standardized patient (SP) displaying a manic episode and another displaying a major depressive episode with psychotic features) with students holding introductory conversations with both patients; and (5) lectures on mental illness and its treatment delivered by nurses working in mental health departments. The authors collected demographic data before the intervention and used a pre- and post-intervention questionnaire to assess the students' future intention of working with the mentally ill and their attitudes toward patients with psychiatric illness, including stigma, perceived dangerousness, and potential for devaluation and discrimination of patients with mental illness. Overall, the intervention led to slight improvements in students' attitudes but did not improve students' intentions to work with patients with mental illness. The perception of the mentally ill as dangerous was an important factor, having a significantly negative correlation with intention to work with psychiatrically ill patients both preand post-intervention. Interestingly, students rated only the simulation with SPs and the professional lectures as interventions that had significant impact. Older students and Jewish students (in comparison with students who were Christians and Muslim Arabs) held more positive attitudes toward the functional characteristics of patients with mental illness. Being older also correlated positively with the perception of the mentally ill as being less dangerous. These interesting results suggest that not all educational experiences and interventions are the same. As the authors recommend, educational programs should focus on reducing fear regarding patients with mental illness, increasing theoretical knowledge, and promoting positive contacts. 
In a study of medical students at Yale University [6] (representing all 4 years, advanced year, and combined programs), 289 students (42\% response rate) received $\$ 20$ to participate in a 42 -item survey that included questions on demographics, attitudes toward people with mental illness, and beliefs about cause of mental illness and perceived efficacy of psychiatric treatment for six conditions: anxiety, depression, bipolar disorder, schizophrenia, diabetes mellitus, and hypertension. The latter part of this questionnaire comprised a subset of questionnaires from other studies. Whether this questionnaire was validated is unclear, which may limit the interpretation of this study's results. The investigators offered the questionnaire twice but not specifically before or after any educational experience. They analyzed results in regards to year in medical school (preclinical, clinical, or advanced), gender, birthplace, personal experience with mental illness (self, family, friend), and previous completion of the psychiatry clerkship. The investigators conducted an exploratory analysis and identified three main factors: social acceptance (related to socializing with mentally ill patients), belief in supernatural causes (which included four items, such as believing in witchcraft or curses as causes of mental illness), and belief in biopsychosocial causes (including biogenetic, psychological, and social causes of mental illness). Students' attitudes were generally positive, with $87 \%$ reporting social acceptance of people with psychiatric illness, $98 \%$ believing in biopsychosocial causation, and $11 \%$ believing in supernatural causes of mental illness. There was no evidence, however, that either more years of medical education or completion of a psychiatric clerkship (6-week inpatient and outpatient experience, team-based, with 3 weeks spent taking care of patients at the medical interface) changed attitudes on any of these three factors. Interestingly, as one would probably predict, students with personal experience of mental illness had higher scores on social acceptance, and students born outside the USA were more likely to believe in supernatural causes of mental illness. Completion of a psychiatry clerkship was associated with the stronger belief that anxiety disorders and diabetes mellitus can be effectively treated, which may be a good sign. These results do not seem to be very encouraging in regards to the impact of the psychiatric educational experience at one medical school.

Finally, researchers at a major medical school in Brazil administered a 56-item questionnaire to 99 medical students before and 142 medical students after a psychiatric clerkship [7]. Corresponding pre- and post-response rates varied from 67 to $86 \%$. Because the questionnaires were administered anonymously, it was impossible to match responses. Similar to the Yale University study [6] (two coauthors of this study were also coauthors of the Yale study), four factors were identified in an exploratory factor analysis: social acceptance of people with mental illness, normalization of the roles for people with mental illness in society, non-belief in supernatural causation of mental illness, and belief in biopsychosocial causes of mental illness. Scores for social acceptance, nonbelief in supernatural causes of mental illness, and belief in biopsychosocial causes of mental illness were generally high before the clerkship. Only social acceptance of people with mental illness, however, improved significantly following the clerkship. As the authors noted, the lack of change in other factors may reflect the ceiling effects in response before the clerkship. It would be interesting and enlightening to know more about the clerkship at this school and the material covered during the clerkship. Knowing more about the clerkship could help us understand why this experience was not as effective as one would wish and whether it should be further investigated and modified with a follow-up study.

What can we conclude from this small international collection of studies examining the impact of educational experience on attitudes toward mental illness? First, educational experiences do have an impact on student views and attitudes toward mental illness. This impact is small at times and varies depending on what factors are examined and what interventions are implemented. We need to know more about the important influential variables that lend to more positive attitudes. Second, it seems that positive and negative attitudes and views of mental illness and people with mental illness are likely similar among many trainees, including medical students, nursing students, and perhaps others. Third, other factorssuch as personal experience with mental illness, age of the student, and cultural background - may influence student attitudes more than their educational experience. In addition, the impact of perceived dangerousness is important and clearly continues to stigmatize patients with mental illness and the attitudes of trainees toward them. Last, but not least, the increases of acceptance of those with mental illness were slight at most in these three studies. Attitudes may be harder to influence than knowledge and skills, because attitudes may have been established many years before medical training. These findings suggest that we must continue not only to destigmatize mental illness as a whole but also to provide our trainees with a realistically positive learning environment and perspective of our field.

The findings of Itzhaki and colleagues [5] on decreased intention to work with mentally ill patients after an educational experience in the field highlight a previous debate on mixing the study of attitudes toward psychiatry with trainees' career choice. As we have argued previously [8], the study of these two must be separated - there is a difference between attitudes and behavior. Our field frequently conflates career choice with attitudes of tolerance and acceptance toward working with psychiatric patients. As Lyons and Janca [9] correctly pointed out, psychiatry rotations (and most other 
educational experiences) are not primarily developed as recruitment tools. Therefore, although they can be effective in improving trainees' attitudes toward psychiatry, evidence of their effect on psychiatry as a career choice is mixed.

At this point, we must create more defined outcome measures of career choice and not simply rely on post-educational experience surveys following educational exposures that question students regarding their attitudes toward psychiatry and their desire to pursue the specialty after graduation. We need to expand this area of study by asking people who have already selected psychiatry as their career what influenced their decision. This study could be done retrospectively, perhaps after the Match, or prospectively, by following students who rated their educational experience as positive. We also need to expand our strategies to overcome stigma toward mental illness and the mental health profession in preclinical teaching to better prepare students for their clerkship [10]. We should reexamine our mission during teaching psychiatry to medical and other students. Is our mission recruitment, or is it acceptance of caring for psychiatric patients in the context of other fields? Both are important, but fostering better knowledge, experience, and acceptance of psychiatry in other specialties will help us humanize medical practice and, at least in part, diminish the shortages we face in caring for people with mental illness. We also need to better integrate psychiatry with other parts of medical school curriculum. Recruitment is certainly also important because there are very few countries where recruitment to psychiatry is not a problem [11].

Although the tide may be changing by bringing more medical students into our specialty, we should not relent in our efforts to understand what drives students' attitudes and views toward our field. We should also continue in our quest to fight the stigma of mental illness and to understand what encourages our earlier career colleagues to become psychiatrists.

\section{Compliance with Ethical Standards}

Disclosures On behalf of all authors, the corresponding author states that there is no conflict of interest.

\section{References}

1. Nielsen 3rd AC, Eaton Jr JS. Medical students' attitudes about psychiatry. Implications for psychiatric recruitment. Arch Gen Psychiatry. 1981;38:1144-54.

2. Balon R, Franchini GR, Freeman PS, Hassenfeld IN, Keshavan MS, Yoder E. Medical students' attitudes and views of psychiatry. 15 years later. Acad Psychiatry. 1999;23:30-6.

3. Lyons Z. Attitudes of medical students toward psychiatry and psychiatry as a career: a systematic review. Acad Psychiatry. 2013;37:150-7.

4. Lyons Z. Impact of the psychiatry clerkship on medical student attitudes toward psychiatry and to psychiatry as a career. Acad Psychiatry. 2014;38:35-42.

5. Itzhaki M, Meridan O, Sagiv-Schifter T, Barnoy S. Nursing students' attitudes and intention to work with mentally ill patients before and after a planned intervention. Acad Psychiatry. 2016. doi:10.1007/s40596-016-0521-3.

6. Chiles C, Stefanovics E, Rosenheck R. Attitudes of students at a US medical school toward mental illness and its cause. Acad Psychiatry. 2016. doi:10.1007/s40596-016-0508-0.

7. da Rocha Neto HG, Rosenheck RA, Stefanovics EA, Cavalcanti M. Attitudes of Brazilian medical students towards psychiatric patients and mental illness: a quantitative study before and after completing the psychiatric clerkship. Acad Psychiatry. 2016. doi:10.1007 /s40596-016-0510-6.

8. Balon R. Does a clerkship in psychiatry affect medical students' attitudes toward psychiatry? Acad Psychiatry. 2008;32:73-5.

9. Lyons Z, Janca A. Towards protecting the endangered discipline of psychiatry. Aust N Z J Psychiatry. 2015;49:388-9.

10. Lyons Z, Janca A. Impact of a psychiatry clerkship on stigma, attitudes towards psychiatry, and psychiatry as a career choice. BMC Med Educ. 2015;15:34.

11. Shields G, Ng R, Ventriglio A, Castaldelli-Maia J, Torales J, Bhugra D. WPA position statement on recruitment in psychiatry. World Psychiatry. 2017;16:113-4. 